\section{Lack of Evidence for Attributing Chlorhexidine as the Main Active Ingredient in Skin Antiseptics Preventing Surgical Site Infections}

To the Editor-We read with great interest the articles by Noorani et al $^{1}$ in the British Journal of Surgery and by Lee et $\mathrm{al}^{2}$ in Infection Control and Hospital Epidemiology. The authors have published almost simultaneously two systematic reviews of what is described as comparisons of chlorhexidine versus povidone-iodine for preoperative skin antisepsis. On the basis of their analyses, the authors conclude that chlorhexidine is the more effective antiseptic in preventing surgical site infections (SSIs). However, we believe that this conclusion is not sufficiently supported by the studies included.

In both articles, the effect of preventing SSIs is solely attributed to chlorhexidine. However, the majority of studies were based on chlorhexidine-alcohol mixtures. When attributing effects to factors, it is necessary to look for other factors that could also be influencing these effects. The common agents for preoperative skin antisepsis are (1) alcohols, (2) chlorhexidine, and (3) povidone-iodine. Both chlorhexidine and povidone-iodine are available in aqueous formulations and in alcoholic formulations. Aqueous formulations have 1 active ingredient, whereas alcoholic formulations include 2 active ingredients. The differential antimicrobial activity of these compounds has been a topic of intense research and evaluation since the 1970s and is well described in infection control textbooks and guidelines. ${ }^{3-6}$ If aqueous chlorhexidine or povidone-iodine is compared with various alcohols, then the immediate antimicrobial activity of the alcohols is significantly greater than that of the aqueous agents, by a factor of about $1 \log$ (ie, a 10-fold difference). Alcohol is clearly a powerful skin antiseptic on its own, and in alcoholic chlorhexidine or povidone-iodine formulations it is the agent that contributes most to the overall activity. We have highlighted this in a previous letter to the editor. ${ }^{7}$ Formulations of alcohols with chlorhexidine or povidone-iodine may have some added activity or persistency, which pure alcohols do not have. ${ }^{6}$

In our opinion, the articles by Noorani et $\mathrm{al}^{1}$ and Lee et $\mathrm{al}^{2}$ have not shown clear and unambiguous evidence that the observed effects are solely or even mainly due to chlorhexidine, despite this being the main claim put forward by the authors. At the same time, they ignore the effects that the alcohol in the antiseptics is likely to have had. Beyond the mere presence of chlorhexidine and povidone-iodine, the studies analyzed in both articles are very heterogeneous. Some compared alcoholic chlorhexidine with aqueous povidoneiodine, one compared aqueous preparations in both study arms, and some compared alcoholic preparations in both arms. Some assessed SSI rates as the outcome, others (analyzed in Lee et $\mathrm{al}^{2}$ ) assessed skin microbial cultures after antisepsis. One recent study with significantly different SSI rates as the outcome, ${ }^{8}$ contributing a large number of observations to the analyses, compared the use of alcoholic chlorhexidine to that of aqueous povidone-iodine, where the alcohol in the chlorhexidine formulation clearly confers an a priori advantage. One study involved vaginal surgery exclusively. The situation for vaginal surgery is fundamentally different from surgery through superficial skin, since the physiology of mucous membranes and the quality and quantity of the microbial flora are vastly different, and only aqueous preparations can be used. One earlier study from 1982 used alcoholic formulations in both arms but did not specify the alcohol concentrations used and whether they were in the antimicrobially active range, which means that no conclusions can be drawn. None of the studies with microbial culture results as the end points used any US or European standardized methods for antiseptic or disinfectant testing. Also, it is important to note that none of these studies specified whether they used any neutralizer substances in the experiments. Neutralizers are essential for some antiseptic testing experiments because some antiseptics continue to kill microorganisms after sampling, so that in the absence of neutralizers falsely higher kill rates will be seen. ${ }^{9}$ Chlorhexidine in particular appears to be prone to this effect, with falsely low colony counts being a consequence. ${ }^{9}$

Our cautious guess is that if there were sufficient data available for a meta-analysis comparing alcoholic with aqueous skin antiseptics, the alcohol-based ones would probably fare very well. Just as well as being a study of chlorhexidine versus povidone-iodine, the article by Darouiche et $\mathrm{al}^{8}$ could have been labeled a study of an alcoholic versus an aqueous compound. Interestingly, a very large recent study using 1 alcoholic chlorhexidine protocol and 2 alcohol-containing iodine preparation protocols, ${ }^{10}$ all with alcohol concentrations in the active range, showed a small but statistically significant advantage of the iodine-containing protocols. We conclude that based on the known properties of these different antiseptics, alcohols should remain the primary component of surgical antiseptics for superficial skin, at least until further evidence becomes available. The efficacy of chlorhexidine versus iodine compounds should be determined under otherwise equivalent conditions, such as in sufficiently powered randomized clinical trials comparing alcoholic formulations. This would ultimately resolve this important clinical question.

\section{ACKNOWLEDGMENTS}

Potential conflicts of interest. All authors report no conflicts of interest relevant to this article. 


\section{Matthias Maiwald, $\mathrm{MD}, \mathrm{PhD}$; \\ Andreas F. Widmer, MD, MS; ${ }^{2}$ Manfred L. Rotter, MD, Dip Bact ${ }^{3}$}

\section{The Importance of Isopropyl Alcohol in Skin Preparation Solutions}

\begin{abstract}
Affiliations: 1. Department of Pathology and Laboratory Medicine, KK Women's and Children's Hospital, Singapore; 2. Division of Infectious Diseases and Hospital Epidemiology, University of Basel Hospitals, Basel, Switzerland; 3. Institute of Hygiene and Applied Immunology, Medical University of Vienna, Vienna, Austria.

Address correspondence to Matthias Maiwald, MD, PhD, Department of Pathology and Laboratory Medicine, KK Women's and Children's Hospital, 100 Bukit Timah Road, Singapore 229899, Singapore (matthias.maiwald@ kkh.com.sg).

Infect Control Hosp Epidemiol 2011;32(4):404-405

(C) 2011 by The Society for Healthcare Epidemiology of America. All rights reserved. 0899-823X/2011/3204-0017\$15.00. DOI: $10.1086 / 659253$
\end{abstract}

\section{REFERENCES}

1. Noorani A, Rabey N, Walsh SR, Davies RJ. Systematic review and meta-analysis of preoperative antisepsis with chlorhexidine versus povidone-iodine in clean-contaminated surgery. Br J Surg 2010;97:1614-1620.

2. Lee I, Agarwal RK, Lee BY, Fishman NO, Umscheid CA. Systematic review and cost analysis comparing use of chlorhexidine with use of iodine for preoperative skin antisepsis to prevent surgical site infection. Infect Control Hosp Epidemiol 2010;31: 1219-1229.

3. Gröschel DHM, Pruett TL. Surgical antisepsis. In: Block SS, ed. Disinfection, Sterilization and Preservation. 4th ed. Philadelphia: Lea \& Febiger, 1991:642-654.

4. Rotter ML. Hand washing, hand disinfection, and skin disinfection. In: Wenzel RP, ed. Prevention and Control of Nosocomial Infections. 3rd ed. Baltimore: Williams \& Wilkins, 1997:691-709.

5. Rotter ML. Hand washing and hand disinfection. In: Mayhall CG, ed. Hospital Epidemiology and Infection Control. 3rd ed. Philadelphia: Lippincott Williams \& Wilkins; 2004:1727-1746.

6. Mangram AJ, Horan TC, Pearson ML, Silver LC, Jarvis WR, Hospital Infection Control Practices Advisory Committee. Guideline for prevention of surgical site infection, 1999. Infect Control Hosp Epidemiol 1999;20:250-278.

7. Maiwald M, Widmer AF, Rotter ML. Chlorhexidine is not the main active ingredient in skin antiseptics that reduce blood culture contamination rates. Infect Control Hosp Epidemiol 2010; 31:1095-1096.

8. Darouiche RO, Wall MJ Jr, Itani KM, et al. Chlorhexidinealcohol versus povidone-iodine for surgical-site antisepsis. $N$ Engl J Med 2010;362:18-26.

9. Kampf $G$, Shaffer $M$, Hunte C. Insufficient neutralization in testing a chlorhexidine-containing ethanol-based hand rub can result in a false positive efficacy assessment. BMC Infect Dis 2005; 5:48.

10. Swenson BR, Hedrick TL, Metzger R, Bonatti H, Pruett TL, Sawyer RG. Effects of preoperative skin preparation on postoperative wound infection rates: a prospective study of 3 skin preparation protocols. Infect Control Hosp Epidemiol 2009;30: 964-971.
To the Editor-We read with great interest the recent article by Lee et $\mathrm{al}^{1}$ comparing chlorhexidine and iodine for skin antisepsis to prevent surgical site infection. We wish to commend the authors for their timely execution and thoughtful approach to this analysis.

We would like to contribute to this discussion by pointing out what we believe to be a very important aspect of the debate: the importance of isopropyl alcohol in solutions. Isopropyl alcohol (IPA) has been shown in both in vitro and in vivo studies to be a powerful and rapid antiseptic agent on the skin. According to many studies and texts on the subject, both chlorhexidine and iodine have an intermediate onset of action and a later peak effect, thus making the combination of immediate action provided by IPA and delayed action in the mixed solutions theoretically superior to chlorhexidine and iodine alone. In their meta-analysis, the authors made no distinction between solutions containing IPA and those that did not contain this important agent when they drew the conclusion that chlorhexidine is superior to iodophores. We present an argument against this conclusion.

First we would like to discuss a large prospective study by our group that compared surgical site infection (SSI) rates during 3 separate 6-month time periods (spanning from January 1,2006 , to June 30,2007 ) during which 3 different skin preparation protocols employing IPA were used for all adult general surgery cases at our institution. ${ }^{2}$ We found that rates of SSIs were significantly decreased when iodophore-based skin preparation solutions containing IPA were used, compared with SSI rates when solutions of chlorhexidine plus IPA were used. Because all preparation protocols included IPA, we could isolate the nonalcohol component (chlorhexidine, iodine-providone, and iodine povacrylex were used in our study), and we concluded that iodine-based solutions were most likely superior to chlorhexidine-based solutions in both intention-to-treat and per-protocol models.

Furthermore, when examining the studies used in the systematic review by Lee et $\mathrm{al}_{,}{ }^{1}$ we see that the authors found only 3 studies (Saltzman et al, ${ }^{3}$ Ostrander et al, ${ }^{4}$ and Veiga et $\mathrm{al}^{5}$ ) that compared iodophore solutions that contain alcohol with chlorhexidine solutions that contain alcohol. In these studies, the outcomes measured were positive skin swab cultures and/or SSIs. In the Saltzman et $\mathrm{al}^{3}$ and Ostrander et $\mathrm{al}^{4}$ studies, there were fewer positive skin swab cultures in the chlorhexidine group, but the SSI rates were not significantly different between the two groups. Only in the study by Veiga et $\mathrm{al}^{5}$ were they able to show a decrease in SSI rates in the chlorhexidine group. With the exception of the studies by Culligan et $\mathrm{al}^{6}$ (which compared 2 non-IPA-containing solutions) and Berry et $\mathrm{al}^{5}$ (which contained a chlorhexidineplus-spirits solution), all of the other studies compared chlorhexidine plus IPA with iodophore agents that did not contain alcohol. We do not believe that this is a valid comparison. 